\title{
Dogs as Sculpture Models: Is IACUC Review Needed?
}

There are times when a complicated problem arises from what seems to be a simple issue. This is what happened to the Institutional Review Board (IRB) at Great Eastern University. (The IRB is roughly the equivalent of the IACUC for research using human subjects.)

Dr. Sandra Gershowitz planned to study the effect of a novel art therapy on clinically depressed persons who also had a significant visual disability. The crux of the concept was to use the person's guide dog as a tactile sculpture model. Gershowitz, a faculty member of the University, was highly respected in the field. She planned to conduct the study in the art therapy room of Great Eastern University Hospital. The hospital was a separate corporation from the University although they were in adjoining buildings. The study was funded by the National Institute of Mental Health.

No significant issues arose at the IRB meeting, except that Dr. Sherry Smith, who was also an IACUC member, suggested that IACUC approval would also be required because of the guide dog. Her comment elicited a chorus of laughs and disbelieving stares from other IRB members, almost none of whom had ever had any interaction with an IACUC. They could not believe that an approval of any kind was needed for a dog that was specifically trained to help a human being and was to do nothing more than sit still and be gently touched while being a model for an art therapy project. The dog was not to be evaluated in any way whatsoever. Furthermore, they said, this therapy was to occur at a hospital that had no formal affiliation with Great Eastern University and its IACUC. Dr. James Stark, who did have some IACUC experience, likened the use of the guide dogs to a study in which snakes were the object of the research, and live neonatal mice were fed to the snakes. He said, "no
IACUC would ever require a protocol and approval for the use of neonatal mice as feed, and the analogy holds true in this study. The dogs are not the object of the study ... the people are the objects. The dogs are totally and completely of no concern.”

Was Smith or Stark right in their assertions? Must an IACUC have to approve the use of the guide dogs in Gershowitz's study?

\section{IACUC-IRB Cooperation Needed}

\section{Harry Fyke, DVM}

This question seems to evoke one of those 'gray zone' cases that compliance boards often encounter. Whether or not it should require IACUC approval may not be as important as ensuring that steps are taken to assure the health and welfare of both the animals and humans involved.

If Great Eastern University has an NIH/OLAW Assurance, the IRB should consult it, because some institutions require approval by the IACUC for all uses of vertebrate animals.

That the research is to take place at an off-site facility is certainly not as important as the fact that this is a PHS-supported project that receives funding through the University. This makes it subject to the oversight of the University. The bottom line here is that this is an NIH-funded research project that involves a USDAcovered species ${ }^{1}$.

The IACUC in collaboration with the IRB, should decide whether the oversight is handled by the IRB or requires IACUC involvement. The discussion may be limited to the respective Chairs and the Attending Veterinarian.

There are certainly occupational health and safety issues that need to be addressed. Service animals are generally screened for health problems, including disposition, while they are being trained, but there are no requirements for the maintenance of their health by private owners. Therefore the potential exists for them to harbor and transmit diseases and/or parasites to other animals, as well as humans. A veterinarian
A Word From OLAW

In response to the questions posed in this scenario, the Office of Laboratory Animal Welfare (OLAW) offers the following clarification and guidance:

This month's scenario describes unusual animal use in a nontraditional research setting. The National Institute of Mental Health is a component of the National Institutes of Health $(\mathrm{NIH})$, a Public Health Service (PHS) agency. As was stated by some of the reviewers this is, by definition, a PHS-supported activity involving live vertebrate animals; therefore, as a condition of funding, the awardee institution and all performance sites must be covered under one or more Assurances on file with OLAW and verification of IACUC approval must be submitted prior to award. The IACUC review criteria and oversight requirements should be specially tailored to address those practical issues (e.g., health and welfare of animals and humans involved) already raised in the responses above.

The scenario also mentions, as a proposed analogy for consideration of the issues involved in the dog study, the practice of feeding live mice to snakes. It should be noted that in situations where the PHS Policy is applicable, the feeding of live vertebrate animals to other animals is also subject to IACUC oversight either as a part of the protocol review process or as a covered component of the institutional program of animal care and use.

Nelson L. Garnett, DVM

Director, Office of Laboratory Animal Welfare

National Institutes of Health 